\title{
Linx
}

Revue des linguistes de l'université Paris X Nanterre

5 | 1994

La négation

\section{La négation et les verbes d'adhérence. Pour en finir avec le neg-raising}

\section{Robert Forest}

\section{OpenEdition}

Journals

Édition électronique

URL : http://journals.openedition.org/linx/1192

DOI : 10.4000/linx.1192

ISSN : 2118-9692

Éditeur

Presses universitaires de Paris Nanterre

\section{Édition imprimée}

Date de publication : 1 juin 1994

Pagination : 49-58

ISSN : 0246-8743

\section{Référence électronique}

Robert Forest, "La négation et les verbes d'adhérence. Pour en finir avec le neg-raising », Linx [En ligne], 5 | 1994, mis en ligne le 18 juillet 2012, consulté le 30 avril 2019. URL : http:// journals.openedition.org/linx/1192; DOI : 10.4000/linx.1192

Ce document a été généré automatiquement le 30 avril 2019.

Département de Sciences du langage, Université Paris Ouest 


\title{
La négation et les verbes d'adhérence. Pour en finir avec le neg-raising
}

\author{
Robert Forest
}

1 Voilà un sujet qui n'est certes pas nouveau. J'y reviens parce que je constate que les noms qui font allusion à quelque chimère transformationaliste ("promotion» ou « avancement » de la négation, neg-raising, transportation, etc.) hantent encore les écrits de linguistes qui en ont parlé récemment, comme Iordanskaja (1986) et surtout Horn (1989 : cf. notamment le sous-chapitre 5.2, pp. 308-330).Je voudrais revenir aussi sur ma propre analyse (Forest, 1983), dont je garde l'essentiel: caractère non syntaxique du problème, régissants sémantiquement « descriptifs » impliquant un « engagement » non performatif, continuum informatif et empathique entre régi et régissant; je souhaite modifier un peu l'éclairage en introduisant, notamment, la notion d'adhérence.

On peut sans doute considérer que l'analyse du phénomène en termes de transformation est morte de sa belle mort. Elle résultait d'un tour de passe-passe (les transformations étaient censées préserver le sens; on voyait des énoncés de sens analogue; on en déduisait qu'ils étaient liés par une relation transformationnelle). Elle se heurtait à diverses difficultés (par exemple, il est difficile de ne pas rapprocher, pour le sens, Je ne veux pas qu'il vienne, équivalent à Je veux qu'il ne vienne pas, de Je ne veux pas de carottes ; mais de quoi ce dernier énoncé est-il la transformation par «transport de négation »?). Rappelons enfin la principale objection interne à l'analyse transformationnelle : il devrait y avoir ambiguïté pour un énoncé tel que Je ne veux pas qu'il vienne, correspondant soit à une négation primitivement incidente à vouloir, soit à une négation "montée " de la subordonnée ; or rien n'apparaît de tel. C'est déjà en substance l'argument de Bartsch (1973). 


\section{1.}

3 Le phénomène translinguistique étudié est essentiellement une équivalence sémantique entre énoncés; je l'appellerai équivalence E. Il s'agit, rappelons-le, de la capacité de certaines expressions prédicatives régissantes à entraîner l'apparition d'une équivalence de sens entre les énoncés négatifs où elles-mêmes sont niées (le régi étant positif) et ceux où leur régi est négatif (elles-mêmes étant positives). Ainsi, en français : Je ne crois pas qu'il pleuve et Je crois qu'il ne pleut pas ;

4 Je précise : $1^{\circ}$ ) Que cette situation a vraisemblablement une vocation universelle dans les langues, même si le spectre des expressions régissantes concernées varie d'une langue à l'autre, et même si certaines langues tendent justement à traiter les expressions du vouloir, du devoir, voire du croire et du sembler comme des morphèmes grammaticaux auxquels la négation ne peut souvent pas être contrastivement incidente, d'où l'absence d'équivalences $\mathrm{E}$.

$52^{\circ}$ ) Que l'équivalence sémantique $\mathrm{E}$ n'est pas forcément une synonymie absolue. Elle ne peut néanmoins manquer de frapper les linguistes par contraste avec le cas d'autres expressions régissantes qui ne produisent absolument pas les mêmes phénomènes (par exemple en français: savoir, aimer, douter, promettre, pouvoir, laisser, voir, se rappeler, persuader, (s')imaginer, et ainsi de suite).

$63^{\circ}$ ) Que je parle d' "expressions régissantes » et de rection en un sens très large; il peut s'agir de subordination, de complémentation de type infinitif, ou d'autres phénomènes encore (le cas échéant, rapport entre un adverbe de phrase et le reste); on peut parfois aussi sauter un maillon (si l'on a un régi intermédiaire comme un équivalent de verbe être , etc.).

7 La démarche que je me suis proposée a d'abord consisté à inventorier et à présenter systématiquement les régissants susceptibles d'entraîner l'équivalence E. Il me semble qu'on peut rapporter tous les cas cités par d'autres ou rencontrés par moi à un tableau à quatre cases. Tous les régissants partagent (et possèdent exclusivement, selon moi) une propriété sémantique dont la dénomination la moins inappropriée me paraît être: adhérence à une maxime.

Adhérence à une maxime :
$\begin{aligned} & \text { épistémique, } \\ & \text { aléthique }\end{aligned}$

Instance extérieure à
l'entité décrite comme
adhérant à la maxime: $\left\{\begin{array}{l}\text { non } \\ \text { oui }\end{array}\right.$

Exemples pour (1) : français croire, penser, considérer, avoir l'impression, anglais think, believe, suppose (au sens de " avoir une opinion »), imagine, berbère tn « admettre l'idée, liée à une apparence, que $»$.

Pour (2) : français vouloir, désirer, avoir l'intention de.

Pour (3) : français sembler, paraître, avoir l'air, donner à penser, on dirait, anglais pretend.

Pour (4) : français il faut, devoir, convenir, avoir à, être censé, anglais be supposed (I’m not 
supposed to go out with boys, chante Marilyn Monroe dans Lolita), allemand sollen (es soll nicht sein, daß/es soll nicht sein, $d a \beta$ nicht).

Et les équivalents plus ou moins approximatifs de ces régissants prototy-piques dans un grand nombre de langues. verbalisation de ce que serait pour quelqu'un le fait de faire tel ou tel acte. J'allège la condition que cela concerne un sujet humain conscient. Puis j'établis une proportion : la maxime qui va avec croire ou sembler est à celle qui convient à vouloir ou devoir ce que l'être est au devoir-être, l'épistémique au pratique, l'état de choses à l'action. On notera que, dans l'analyse contrastée du croire et du vouloir, du sembler et du devoir, j'indicie non le régissant, mais le régi (vu comme référant à une maxime) du caractère ontique ou déontique. Cela ne me paraît pas contre-intuitif. J'entends qu'une maxime puisse se prêter à un jugement en termes de vrai ou de faux dans le premier cas, de bon ou de mauvais dans le second cas («bon », au sens de « à faire », comme dans il serait bon que).

11 Je soutiens maintenant que pour que l'équivalence $\mathrm{E}$ puisse se produire, l'entité décrite (qu'une instance extérieure à elle intervienne en sus ou non) doit être décrite comme en état d'adhérence, ni plus ni moins, à la maxime considérée. Attention : diverses traditions philosophiques plus ou moins bien comprises (notamment l'idée de croyances et de volontés entreposées dans quelque "amère, sombre et sonore citerne ») font que nous répugnons à concevoir - ou à faire concevoir à ce qu'on appelle " psychologie populaire " - le «croire», le "vouloir», etc., comme n'impliquant pas le choix d'une "attitude propositionnelle ». Mais ce n'est pas à une adhésion, c'est à une adhérence, à un collage, que renvoient apparemment les langues.

\section{2.} choses.

\section{$1^{\circ}$}

Régler un certain nombre de cas-limites, entraînant sans doute aucun l'équivalence en question, mais pas très faciles à classer.

14 Ainsi avoir l'habitude de et ses équivalents concernent la pratique ; nous sommes donc dans la case $n^{\circ} 2$, même s'il n'y a pas l'idée de « volonté ». 
15 L'italien non fare « empêcher, faire en sorte [par sa seule existence] que ne pas » est sans doute une métaphore d'intentionnalité à ranger sous le $\mathrm{n}^{\circ} 2$. L'exemple Il caffe non $\mathrm{mi}$ fa dormire laissait Horn insomniaque. Dans ce passage de Iacopone da Todi (XIIIe siècle), la mère de Jésus dit à Pilate :

o Pilato, non fare

Il figlio mio tormentare

Est-ce «s'abstenir de faire» ou «empêcher, faire en sorte que ne pas»? On voit justement que les deux se rejoignent - et voilà l'équivalence $\mathrm{E}$ in statu nascendi - pour peu qu'il y ait adhérence, c'est-à-dire que Pilate soit décrit, non comme maître du choix de son attitude, mais plutôt comme non-maître de ne pas faire intervenir sa volonté parce qu'engagé volens nolens dans des rapports tels que la maxime pratique («que quelqu'un torture Jésus») soir nécessairement bonne (à faire) ou mauvaise (à ne pas faire). Autrement dit, si Pilate ne peut pas « s'en laver les mains », l'équivalence E apparaît.

Le cas du grec ancien ou phemi est assez délicat. Il doit y en avoir deux sortes. Le premier, avec marque négative accentuée, veut sans doute dire "dire 'non'", d'où " nier ». Le second, avec ou atone, relève sans doute de l'équivalence $\mathrm{E}$ dans la mesure où il signifie "avoir une opinion» (le fait de l'expression de l'opinion étant secondaire). Donc Sophocle, Ajax, 1326, ou phes' eásein « ne pas / il dit / laisser (infinitif futur) » ne veut pas dire « il s'abstient de dire » mais « il n'est pas d'avis qu'il faille permettre ».

Selon les langues, on fait souvent figurer parmi les prétendus neg-raising triggers des verbes qui paraissent avoir une valeur active et, plus ou moins nettement, performative ; ainsi conseiller en français (je ne te conseille pas de me marcher sur les pieds - (?) je te conseille de ne pas me marcher sur les pieds); en grec ancien sumbouleúo « je conseille» ou keleúo « je conseille (vivement), je pousse à, j'ordonne »; russe velet' " ordonner », etc. Or il est clair que, lorsque ces verbes ont un sens pleinement actif et performatif, l'effet d'équivalence ne se produit pas : à savoir, si la phrase niant le verbe en question s'interprète comme « je ne te donne pas le conseil / l'ordre de ». En revanche, l'équivalence E se manifeste d'autant plus nettement que les régissants se rapprochent d'un équivalent de vouloir; l'attitude réceptive ou non du second actant n'a aucune pertinence dans ce cas. On retrouve la case $n^{\circ} 2$ du tableau. Notons au passage que les verbes comme vouloir ne sont pas, à l'inverse, sans affinité avec les discours qui conseillent ou ordonnent (cf. le vouloir de précepte, par exemple, dans l'Emile: Je veux qu'on l'habitue à voir des objets nouveaux, des animaux laids...).

\section{$2^{\circ}$}

Analyser les régissants qui n'entraînent l'équivalence E que dans certains contextes. On vient d'en voir un ou deux exemples. Pensons encore au français se soucier, avoir cure, avoir garde; il s'agit certes d'emplois très contraints. Mais il me semble qu'on peut largement assimiler au type $\mathrm{n}^{\circ} 2$ l'emploi de Je ne me soucie pas de réveiller ce genre de fantasme si cela veut dire, en gros, "je ne veux pas", sans que le «souci » soit à prendre au pied de la lettre.

Vouloir lui-même joue parfois des tours. De Cornulier (1973) cite, au conditionnel, l'absence de «quasi-synonymie » entre Je ne voudrais pas être Dieu et Je voudrais ne pas être Dieu, le second énoncé présupposant selon lui « je suis Dieu » et le premier « je ne suis pas Dieu ». Il est clair que dans le second cas on a affaire à un emploi formulaire de je voudrais qui veut dire à peu près «je formule le vœu de », et qui ne peut guère se nier. Les deux 
occurrences de voudrais dans les phrases citées ne sont donc pas comparables, et les chichis de veille ou de lendemain d'apothéose ne s'émettent pas sur la même longueur d'onde. En revanche, on peut fort bien concevoir, un jour de distribution des rôles au théâtre, mettons pour jouer le «Prologue au ciel» de Faust, le refus poli d'un acteur, indifféremment je ne voudrais pas / je voudrais ne pas être Dieu cette saison. On retombe dans le cas $n^{\circ} 2$ ci-dessus.

\section{$3^{\circ}$}

Comparer les langues (ou les états successifs d'une langue) pour voir si des termes réputés équivalents entraînent ou n'entraînent pas l'équivalence $\mathrm{E}$.

Il s'avère généralement que dans les langues où l'on signale qu'un équivalent de « penser » ou de « croire » n'entraîne pas l'équivalence E, c'est qu'en fait on s'éloigne du sémantisme de pure et simple adhérence à une maxime, le régissant signifiant en réalité « avoir présent à l'esprit » ou « être confiant dans le fait que ».

En français classique, on trouve des emplois de il faut qui n'entraînent pas l'équivalence $\mathrm{E}$, ainsi chez Pascal: Il ne faut pas que l'univers entier s'arme pour l'écraser: une vapeur, une goutte d'eau suffit pour le tuer. Cela veut dire « il n'est pas nécessaire que» (cf. l'allemand nicht müssen) et il n'y a pas de rapport à une " maxime ».

Dans plusieurs langues, des équivalents du français espérer donnent lieu à l'équivalence $\mathrm{E}$, ce qui surprend. Mais on voit qu'alors il ne s'agit pas d'un espoir, simplement d'un « s'attendre à » qui est un locataire de la case $\mathrm{n}^{\circ} 1$ : latin non spero « je ne m'attends pas à ce que "; anglais (exemple de Horn) I never saw a purple cow / I never hope to see one; allemand ich will nicht hoffen, daß (à peu près « je ne veux pas croire que, je refuse de m'attendre à ce que »), le sens de «s'attendre à " pour hoffen (par ailleurs, " espérer ») étant attesté par exemple dans Stifter : Er erschrak, da er uns sah, weil er hier keine Menschen $z u$ finden gehofft hatte.

En anglais, pretend, habituellement glosé en français «faire semblant, faire comme si », donne lieu à l'équivalence $\mathrm{E}$; Horn cite ainsi he didn't pretend to understand comme équivalent à he pretended not to understand. Ceci toutefois implique pour pretend un sens moins actif que « faire semblant »; c'est plutôt « il n'avait pas l'air de / il avait l'air de ne pas comprendre ».

\section{$4^{\circ}$}

Enfin, naturellement, voir quels régissants n'entraînent pas l'équivalence $\mathrm{E}$, alors même qu'on pourrait à la rigueur s'y attendre.

On exclut ainsi les verbes qui impliquent une activité, en particulier un choix d'attitude. Ainsi le voudrais cité plus haut. En hindi, ca:hna: «vouloir» entraîne E si le régi est actanciellement coréférent du régissant (« vouloir faire ») et n'entraine pas E dans le cas contraire ("vouloir qu'autrui fasse»). Sans doute parce que le second cas implique la médiation d'une attitude visant à influer sur le cours des choses.

On exclut aussi tous les termes empathiquement marqués, soit positivement (comme espérer, être sûr, être convaincu) soit négativement (douter, refuser, pourtant à peu près équivalents à croire que ne pas, vouloir que ne pas, donc variétés de croire et de vouloir) ; c'est sans doute ce que veut dire Iordanskaja (1986) quand elle parle de «condition de 
non-présupposition » pour obtenir l'équivalence $\mathrm{E}$ : le contact avec une maxime est pour ainsi dire présupposé dans le cas de douter, espérer, refuser, etc., et il s'y ajoute une appréciativité, revenant en fait à un choix d'attitude.

\section{3.}

Après avoir précisé quelque peu les contours de la notion d'adhérence à une maxime, il nous faut maintenant essayer d'expliquer la possibilité et la nécessité de l'équivalence sémantique $\mathrm{E}$ avec ces régissants-là.

Une chose frappe, dont il faut sans doute partir : c'est le caractère auto-hyperonymique des régissants concernés. Croire, vouloir, etc., servent d'hyperonymes aux ensembles de référenciation contenant deux termes complémentaires : croire et son " contraire ", pour ainsi dire, vouloir et son « contraire », etc. Je passe ici de l'analyse lexicale à celle des types d'opérande de la négation, toujours d'un point de vue sémantico-référentiel. Or on a ici affaire, par exemple dans le cas de croire qui vaut pour toute la série, à des opérandes bipolaires où le terme positif et l'hyperonyme qui englobe les deux pôles sont un seul et même terme :

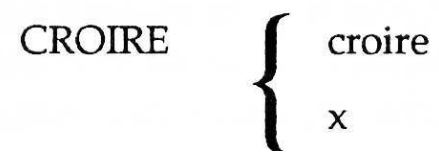
la longueur, de même, croire et son opposé ressortissent au croire. C'est la même idée que l'idée d'adhérence: on ne peut pas ne pas croire (etc.) à partir du moment où on est, sponte sua ou par la médiation d'une instance extérieure, en rapport de contact avec une maxime.

Mais qu'est-ce que $\mathrm{x}$, l'opposé de croire ? C'est le terme sémantico-référentiel auquel nous fera accéder la négation de croire. Est-ce un lexème du type de douter? En un sens, oui, mais l'existence de douter est contingente, tous les locuteurs ne l'emploient pas couramment, et surtout, douter ajoute tout un pan de sens : c'est le côté empathiquement ou appréciativement négatif, le côté apotropaïque, pourrait-on dire. Douter ne fait pas partie du même opérande que croire, puisqu'il implique autre chose que l'adhérence. Le complémentaire de croire (ici on exclut une tierce position, ce qui nous éloigne des longueurs, qui peuvent inclure du ni long ni court) n'a pas d'autre expression linguistique que ne pas croire. Cette expression se compose de la marque négative et du croire hyperonyme (non du croire hyponyme positif).

Passons de l'autre côté. Nos verbes impliquent l'adhérence à une maxime, avons-nous dit, indiciée pour le vrai/faux ou pour le bon/mauvais, c'est selon. Or vrai et faux, bon et mauvais (attention, il ne s'agit pas ici d'une appréciation scalaire qui laisserait ouverte la possibilité d'un ni bon ni mauvais) constituent des ensembles bipolaires de référenciation. Dans les emplois auxquels je pense ici, nier être vrai fait accéder au complémentaire, faux, et nier bon fait retomber sur mauvais.

Introduire une négation dans le régi des expression ici étudiées, c'est donc faire que la maxime, par exemple, de "vraie » qu'elle était, soit maintenant indiciée "fausse », etc. On peut exprimer cela dans des formules dont on n'a pas relevé l'aspect « neg-raiseux » : 
Il n'est pas vrai que $\mathrm{p}=$ Il est vrai que non-p

Il n'est pas bon que $\mathrm{p}=\mathrm{Il}$ est bon que non-p

Mais qu'implique l'obligation de contenu liée à la notion d'adhérence à une maxime? Qu'il y ait impossibilité absolue de dissocier le jeu de l'ensemble de référenciation (croire/ ne pas croire, avec pour hyperonyme croire) du jeu de l'ensemble de référenciation de l'indice approprié de la maxime (vrai/faux, etc.). Cette rigidité fait que, par exemple, si l'on accède pour une raison ou une autre au pôle ne pas croire du premier domaine, on accède ipso facto au pôle faux ou mauvais dans le second domaine. Si l'on accède au pôle faux ou mauvais dans le second domaine, par exemple en niant le régi concerné, on accède ipso facto dans le premier domaine à ce qui ne trouve son expression linguistique adéquate que dans ne pas croire (mais on n'a pas besoin de nier croire puisque cela va de soi). S'il y a deux négations, ne pas croire que ne pas fera revenir au croire, la maxime étant réindiciée "vraie ». Mais ne nous occupons pas de cela : il est suffisamment établi que régissant négatif, régi positif est équivalent pour le sens à régissant positif, régi négatif. Et cela ne peut être le cas que parce que la notion d'adhérence à une maxime produit ce lien rigide entre le jeu des indices des maximes impliqués par les régis, du point de vue de l'intervention diversement incidente de la négation.

37 L'idée de Horn, que les pseudo-neg-raising triggers, répondent à une condition sémantique « du milieu de l'échelle» (mid-scalar condition) - par exemple, croire est intermédiaire entre douter et être sûr - répond sans doute à l'idée que ne pas croire est encore un croire, parce qu'une fois l'échelle retournée, le milieu reste le milieu. Mais Horn ne nous dit pas pourquoi le ne pas croire est un croire que ne pas.

\section{4.}

C'est à ce point sans doute qu'on attend des considérations plus pragmatiques : sur la base de l'équivalence sémantique $\mathrm{E}$, peut-on dégager les conditions du recours préférentiel à la négation du régissant ou à la négation du régi ? Je pense qu'un certain nombre d'options pour l'un ou l'autre relèvent de l'informativité, $\mathrm{du}$ «dynamisme communicatif» (en termes praguois). Par exemple, si l'on pose une question du type Que voulez-vous? il est plus naturel de répondre par Je veux que par Je ne veux pas.

Horn signale que dans certains cas, surtout lorsque (les langues s'y prêtant) le régi comporte un infinitif, la phrase à régi négatif et régissant positif n'est guère attestée. On ne dit guère il veut ne pas venir, il caffe mi fa non dormir, etc. Je ne pense pas que ce constat soit crucial. Le régi négatif n'est pas absolument exclu avec les verbes mentionnés, c'est même en français la seule possibilité avec vouloir à l'impératif : Veuillez ne pas faire de bruit après dix heures. Peut-être les verbes qui admettent fréquemment un complément nominal donnent-ils lieu à des habitudes énonciatives impliquant leur propre négation : Il ne veut pas venir parce qu'on dit souvent Il ne veut pas d'argent. On peut remarquer également que la difficulté d'avoir un régi infinitif nié concerne surtout les cas où la maxime est pratique et non épistémique (voir aussi le peu de naturel de Tu dois ne pas le faire). Dès lors, ne pas venir étant la négation d'un procès actif (indépendamment de l'indice de la maxime) ne s'admet guère sans l'ajout d'un trait statif-descriptif dans l'opérande; ce trait, vu le 
contexte, ne pourrait que finir par revenir à s'abstenir de venir, avec une nuance de volition qui ferait double emploi avec celui qui est mentionné ou impliqué dans le régissant.

Ensuite, ce qui me parait être un serpent de mer pragmatique : toute une littérature, dont Horn se fait l'écho, cherche à montrer que Je ne crois pas qu'il pleuve est "atténué », « plus doux ", litotique, par rapport à Je crois qu'il ne pleut pas.

D'abord, c'est très flou. L'évaluation de la « force » plus ou moins grande ne répond pas à des critères précis. En utilisant le terme vague d' "euphémistique », Horn établit un lien avec sa conception « du milieu de l'échelle ». C'est confusionniste.

Admettons qu'on ait un effet de l'auto-hyperonymie. Je crois que ne pas...est un croire, terme positif, plus marqué peut-être que le croire hypernonymique qu'on a dans Je ne crois pas que... Mais par où passerait, concrètement, l'expression d'une différence entre ces deux croire? Peut-être par une accentuation emphatique, possible sur le seul croire positif; mais le problème est qu'un croire emphatisé cesse d'être un pur verbe d'adhérence et tend à être un autre ensemble lexical, ne donnant justement pas lieu à l'équivalence $\mathrm{E}$.

44 Il me semble que Horn confond la différence de force supposée entre termes liés par l'équivalence $\mathrm{E}$ et la litote. Je ne crois pas que est "euphémistique", sans doute, par rapport à Je suis sûr que ne pas; mais on ne saurait dire cet énoncé euphémistique par rapport à Je crois que ne pas. Il y a sans doute, dans les langues, des habitus énonciatifs idiosyncra-siques, qui peuvent relever de la litote : on dit souvent Je ne crois pas, poliment, dans la conversation. C'est sans doute une litote par rapport à, mettons, Il est absolument évident que ne pas. Mais cela ne préjuge pas de sa «faiblesse » par rapport à Je crois que ne pas, qu'un autre habitus réserverait plutôt à la réponse à une question portant sur les croyances du sujet. Un lien linguistique ne paraît pas pouvoir être établi avec l'équivalence située sur le plan sémantique.

\section{BIBLIOGRAPHIE}

Bartsch, Renate, 1973, „'Negative Transportation' gibt es nicht“, Linguistische Berichte 23.

Cornulier, Benoît de, 1973, «Sur une règle de déplacement de la négation », Le français moderne, 41, pp. 43-57.

Forest, Robert, 1983, " 'Négation promue', insalurité, performatifs et empathie », Bulletin de la Société de Linguistique de Paris, 77-1, pp. 77-97.

Horn, Laurence R., 1989, A Natural history of negation, Chicago et Londres, The University of Chicago Press.

Iordanskaja, Lidija, 1986, « Propriétés sémantiques des verbes promoteurs de la négation en français », Linguisticae Investigationes, 10-2, pp. 345-380. 


\section{RÉSUMÉS}

La transformation de neg-raising n'existe pas, mais il reste à expliquer l'équivalence sémantique constatée entre paires d'énoncés (l'un à régissant nié, l'autre à négation dans le régi) que la théorie du déplacement a exhibées sans convaincre. On défend l'idée que les verbes entraînant l'équivalence en question, dont les quatre catégories sont symbolisées par croire, vouloir, sembler et devoir, se caractérisent comme exprimant l'adhérence (non l'adhésion) à une maxime épistémique ou pratique. Deux propriétés de ces verbes, l'auto-hyperonymie et la solidarité rigide avec les valeurs du régi (vrai/faux, bon/mauvais), expliquent l'effet sémantique lié à la présence de négations.

There is no such thing as neg-raising transformation, but the semantic equivalencies between utterance pairs (one with main verb negated, one with negative subordinate) misdealt with by the raising theory still call for an explanation. It is argued that all four categories of verbs triggering these equivalencies - epitomized with think, want, seem, and have to - should be characterized as expressing adhering (sticking, not giving allegiance) to a maxim, either epistemic or practical. Two properties of verbs of adhering: auto-hyperonymy and rigid attachment to the binary values, true/false and right/wrong, of the complement, explain the semantic effect in contact with negation. 